\title{
Dose Estimates of Occupational Radiation Exposure During Radioguided Surgery of [99mTc]Tc-PSMA- labeled Lymph Nodes in Recurrent Prostate Cancer
}

\author{
Daniel Schmidt ( $\square$ daniel.schmidt@ukr.de) \\ Department of Nuclear Medicine, University Hospital Regensburg \\ Jirka Grosse
}

Department of Nuclear Medicine, University Hospital Regensburg https://orcid.org/0000-0002-91300804

\section{Jutta Moosbauer}

Department of Nuclear Medicine, University Hospital Regensburg

\section{Roman Mayr}

Department of Urology, Caritas St. Josef Medical Centre, University of Regensburg

\section{Maximilian Burger}

Department of Urology, Caritas St. Josef Medical Centre, University of Regensburg

\section{Dirk Hellwig}

Department of Nuclear Medicine, University Hospital Regensburg

\section{Original research}

Keywords: Prostate-specific membrane antigen, prostate cancer, radioguided surgery, theragnostic, radiation protection

Posted Date: December 4th, 2020

DOI: https://doi.org/10.21203/rs.3.rs-117770/v1

License: (a) (1) This work is licensed under a Creative Commons Attribution 4.0 International License. Read Full License 


\section{Dose estimates of occupational radiation exposure during radioguided surgery of [99m Tc]Tc-PSMA-labeled lymph nodes in recurrent prostate cancer}

Daniel Schmidt ${ }^{1}$, Jirka Grosse ${ }^{1}$, Jutta Moosbauer ${ }^{1}$, Roman Mayr $^{2}$, Max Burger ${ }^{2}$, Dirk Hellwig ${ }^{1}$

1 Department of Nuclear Medicine, University Hospital Regensburg, Regensburg, Germany 2 Department of Urology, Caritas St. Josef Medical Centre, University Regensburg, Regensburg, Germany

Keywords: Prostate-specific membrane antigen, prostate cancer, radioguided surgery, theragnostic, radiation protection

Acknowledgment: The authors are grateful to Mr. Goller from the Bavarian State Department of Environment and Radiation Protection (Landesamt für Umwelt, LfU, Kulmbach, Germany) for the discussion of radiation protection issues.

${ }^{\#}$ Corresponding Author

Dr. rer. nat. Daniel Schmidt

Department of Nuclear Medicine

University Hospital Regensburg

D-93042 Regensburg

Germany

Phone: +49 (0) 9419447510

Fax: +49 (0) 9419447502

Email: daniel.schmidt@ukr.de 


\section{ABSTRACT}

\section{Background and objective}

[ $\left.{ }^{99 \mathrm{~m}} \mathrm{Tc}\right]$ Tc-PSMA-based radioguided surgery (TPRS) represents a curative approach for localized relapse of prostate cancer. For its simplified regulatory permission, the radiation protection authorities require a ${ }^{99} \mathrm{mc}$ activity below the exemption limit of $10 \mathrm{MBq}$ at the time of surgery. Our aim was to determine the optimal amount of radioactivity (OAR) to comply with that limit and to estimate the maximum number of TPRS procedures per year and surgeon without triggering the full monitoring obligations.

\section{Methods}

In this retrospective study, a dose rate meter was calibrated using measurements on phantoms and from recently injected ( 1 min p.i.) patients to determine the activity in the patient from measured dose rates. The effective half-life of $\left[{ }^{99 \mathrm{~m}} \mathrm{Tc}\right] \mathrm{Tc}-\mathrm{PSMA}-\mathrm{I} \& \mathrm{~S}$ in patients was determined from repeated dose rate measurements up to $27 \mathrm{~h}$ p.i. to estimate dose parameters of relevance for radiation protection. External exposures of the surgeons were measured with personal dosimeters calibrated in $\mathrm{Hp}(10)$.

\section{Results}

From the first 6 subsequent patients, an effective half-life of $4.15 \mathrm{~h}$ was observed. Assuming an operation time $24 \mathrm{~h}$ p.i., the OAR was $550 \mathrm{MBq}$. Operations lasting in average $2 \mathrm{~h}$ in a distance of $0.25 \mathrm{~m}$ to the patient imply a body dose for surgeons of $4,16 \mu \mathrm{Sv}$ per procedure. Based on these estimates, the surgeon's $\mathrm{Hp}(10)$ is less than $1 \mathrm{mSv}$ per year with up to 241 operations per year. The effective dose for surgeons during the procedure determined with an electronic dosimeter is $4 \pm 1$ $\mu \mathrm{Sv}$.

\section{Summary}

All radiation protection regulations are met with adherence to OAR recommended here without triggering the full monitoring obligations from radiation protection regulations. 


\section{INTRODUCTION}

Prostate cancer ( $\mathrm{PCa}$ ) is the most frequent cancer in men with African ancestry as one main risk factor as well as population ageing, obesity and physical inactivity resulting in a yet increasing incidence [1, 2]. The multidisciplinary therapeutic concept consists of surgery, radiotherapy, androgen deprivation therapy, and chemotherapy [3]. The incidence of a tumor recurring after primary curative therapy is up to $50 \%$. Early detection of a tumor recurrence is crucial for a second attempt of curation. Improvements in mortality of prostate cancer are mainly attributed to the effective treatment of localized lesions which are nowadays detected at increasingly earlier stages [2].

Through the development of radioactively labelled ligands for the prostate specific membrane antigen (PSMA), nuclear medicine offers very specific imaging and therapeutic procedures for the treatment of PCa. These include diagnostic applications using $\left[{ }^{68} \mathrm{Ga}\right] \mathrm{Ga}-\mathrm{PSMA}-11$ or $\left[{ }^{18} \mathrm{~F}\right] \mathrm{F}-$ PSMA-1007 for positron emission tomography/computed tomography (PET/CT) [4] or [ $\left.{ }^{99 \mathrm{~m}} \mathrm{Tc}\right] \mathrm{Tc}-$ labelled PSMA-ligands for single photon emission computed tomography (SPECT)/CT imaging [5], as well as treatment options with [ $\left.{ }^{177} \mathrm{Lu}\right] \mathrm{Lu}-177-P S M A-617$ and $\left[{ }^{225} \mathrm{Ac}\right] \mathrm{Ac}-\mathrm{PSMA}-617$ [6, 7]. A current innovative interdisciplinary approach is the intraoperative localization of recurrent $\mathrm{PCa}$ by $\left.{ }^{99 \mathrm{~m}} \mathrm{Tc}\right] \mathrm{Tc}-\mathrm{PSMA}$-based radioguided surgery (TPRS). PSMA-positive tumor manifestations labelled with $\left[{ }^{99 \mathrm{~m}} \mathrm{Tc}\right] \mathrm{Tc}-\mathrm{PSMA}-\mathrm{I} \& \mathrm{~S}$ are amenable to SPECT/CT imaging and intraoperative detection using a gamma probe. During salvage surgery, PSMA-positive tumor manifestations can be intraoperatively found using a gamma probe and surgically removed even in cases with small or atypically localized lesions $[5,8]$.

According to the German Radiation Protection Act (StrlSchG), the overall responsibility under radiation protection law for the procedure lies with the authorized nuclear medicine physician as the board-certified expert in radiation protection. The prerequisites for the safe handling of ionizing radiation must be established according to the legal requirements. To assure this, estimates of the radiation dose levels and of the amounts of radioactivity have to be known for the different steps during the handling of unsealed radionuclides.

For radioguided surgery of sentinel lymph nodes, a simplified regulatory permission without the need for continuous radiation monitoring of all personal can be obtained in Germany. Therefore, the radiation protection authorities require a ${ }^{99 \mathrm{~m}} \mathrm{Tc}$ activity below the exemption limit of $10 \mathrm{MBq}$ at the time of surgery and the surgeons' whole-body dose must certainly not exceed a total of $1 \mathrm{mSv}$ 
per year. The same conditions apply for a simplified regulatory permission for TPRS. As far as we know, there are no published data for the TPRS on relevant radiation protection parameters, in particular not on the effective half-life of intravenously injected [ $\left.{ }^{99 \mathrm{~m}} \mathrm{Tc}\right] \mathrm{Tc}-\mathrm{PSMA}-\mathrm{I} \& S$, not on the optimal activity to be applied, nor on the radiation exposure of the involved personal.

Thus, the aim of our analysis was to assess the unknown parameters by measurements and to estimate the radiation exposure for involved health professionals as well as the maximum number of TPRS procedures per year and surgeon without triggering the full monitoring obligations. 


\section{MATERIALS AND METHODS}

\section{Patients}

In this retrospective analysis, we included the first subsequent 8 patients who underwent [ $\left.{ }^{99 \mathrm{~m}} \mathrm{Tc}\right] \mathrm{Tc}-$ PSMA-I\&S-SPECT/CT between 11/2018 and 06/2019. Inclusion criterion was suspected localized recurrence from histological proven PCa as detected by $\left[{ }^{68} \mathrm{Ga}\right] \mathrm{Ga}-\mathrm{PSMA}-11 \mathrm{PET} / \mathrm{CT}$ or $\left[{ }^{18} \mathrm{~F}\right] \mathrm{AlF}-$ PSMA-11 PET/CT with intended surgical resection as indicated by the interdisciplinary urooncological tumor board. All patients were referred by urologists to the Department of Nuclear Medicine at the University Hospital Regensburg. The median prostate-specific antigen (PSA) value at the time of imaging was $0,84 \mathrm{ng} / \mathrm{ml}$ (range 0,19 to $34,00 \mathrm{ng} / \mathrm{ml}$ ).

\section{Procedure and Imaging}

[ $\left.{ }^{99 \mathrm{~m}} \mathrm{Tc}\right] \mathrm{Tc}-\mathrm{PSMA}-\mathrm{I} \& \mathrm{~S}$ [9] was freshly prepared and quality-checked in the framework of the German Drug Act (AMG, §13.2b and §55) after notification to the legal authority (Government of Oberfranken, Germany). A mean activity of $684 \pm 29 \mathrm{MBq}$ was intravenously administered. After tracer injection in the morning hours, the patients left the department and returned approximately $2 \mathrm{~h}$ later. All patients underwent whole-body scintigraphy (LEHR collimation, pixel size 2,66 mm, scan speed $12 \mathrm{~cm} / \mathrm{min}$ ) and SPECT of abdomen and pelvis (LEHR collimation, $128 \times 128$ matrix, 128 projections over $360^{\circ}$, 64 stops at 2 projections, dwell time of 15 s per stop) with non-contrast enhanced (low-dose) CT. The low-dose CT was used for attenuation correction and anatomic localization. SPECT/CT was performed $5 \mathrm{~h}$ post injection (p.i.) on a SIEMENS Intevo Bold SPECT/CT system (Siemens Healthineers, Erlangen, Germany). The low-dose CT was acquired with $110 \mathrm{kVp}$ and $50 \mathrm{~mA}$ with a matrix size of $512 \times 512$.

\section{Image analysis}

Images were visually interpreted by 2 experienced nuclear medicine physicians who were also board-certified for CT reading. Physiological uptake in the kidneys, lacrimal and salivary glands, thyroid, liver, gall bladder, spleen, pancreas, digestive tract, kidneys, ureters, and urinary bladder was ignored. Focal uptake that could not be explained by physiological uptake or urinary excretion was interpreted suspicious of prostate cancer (PSMA-positive) [10]. Findings were classified in consensus. In cases where during primary reading at the end of the scan indeterminate results (e.g., tumor uptake vs. tracer excretion in the urinary tract) were present, additional imaging at the next 
day was performed. Figure 1 shows an example of a $\left[{ }^{99 \mathrm{~m}} \mathrm{Tc}\right] \mathrm{Tc}-\mathrm{PSMA}-\mathrm{I} \& \mathrm{~S}$ SPECT/CT compared to a $\left[{ }^{68} \mathrm{Ga}\right] \mathrm{Ga}$-PSMA-11 PET/CT. The PSMA-positive lesion in a PET/CT scan is one of the preconditions of the TPRS and used for cross validation.

\section{Dose rate measurements and calibration for radioactivity levels}

To estimate the amount of radioactivity in the patient as a function of time, dose rate measurements were carried out with a calibratable handheld dose rate meter (RadEye G10, Thermo Fisher Scientific Inc., Waltham, USA). It consists of an energy-compensated Geiger-Mueller tube with a measurement range of 0.5 to $100 \mathrm{mSv} / \mathrm{h}$ and an energy range of $50 \mathrm{keV}$ to $3 \mathrm{MeV}$. A calibration factor was determined for this instrument to calculate the activity in the patient from measured dose rates. Therefore, repeated measurements were performed at $1 \mathrm{~m}$ distance on a cylindrical phantom (diameter $20 \mathrm{~cm}$, height $20 \mathrm{~cm}$ ) filled with ${ }^{99 \mathrm{~m}} \mathrm{Tc}$ (starting activity $667 \mathrm{MBq}$, 8 measurement points measured over 7.1 hours).

The measurement error of the calibration factor $K$ was estimated using the Gaussian error propagation law (GEPL) under the assumption of uncertainties in the post-injection timing of the measurement $\mathrm{t}$, in the amount of the applied activity $\mathrm{A}$ and in the measurement of the dose rate $\mathrm{DR}$, which is mainly influenced by the distance between the patient and the dose rate meter.

From the relation

the GEPL gives

$$
A\left(A_{0}, \lambda, t\right)=A_{0} \cdot \mathrm{e}^{-\lambda \cdot t}
$$

$$
s_{A\left(A_{0}, \lambda, t\right)}=\sqrt{\left(\frac{\partial A\left(A_{0}, \lambda, t\right)}{\partial A_{0}}\right)^{2} \cdot s_{A_{0}}^{2}+\left(\frac{\partial A\left(A_{0}, \lambda, t\right)}{\partial \lambda}\right)^{2} \cdot s_{\lambda}^{2}+\left(\frac{\partial A\left(A_{0}, \lambda, t\right)}{\partial t}\right)^{2} \cdot s_{t}^{2}}
$$

For the parameters $\mathrm{t}, \mathrm{A}$ and $\mathrm{DR}$ our respective assumptions are $\mathrm{s}_{\mathrm{t}}=0.03 \mathrm{~h}, \mathrm{~s}_{\mathrm{A}}=5 \%$ and

$\mathrm{S}_{\mathrm{DR}}=0.3 \frac{\mu \mathrm{Sv}}{\mathrm{h} \cdot \mathrm{MBq}}$. To validate the model, dose rates of recently injected patients were measured from ventrally at a distance of $1 \mathrm{~m}$ at the time of $1 \mathrm{~min}$ p.i. The dose rate measurements were repeated at the time of imaging 2-3 h p.i. and at the time of surgery $20-27 \mathrm{~h}$ p.i.

\section{Estimating the effective half-life and the time to reach the exemption limit}

The time-activity curve of $\left[{ }^{99 m} \mathrm{Tc}\right] \mathrm{Tc}-\mathrm{PSMA}-\mathrm{I} \& \mathrm{~S}$ in the patients was calculated from these dose rate measurements with the calibration factor and fitted to a mono-exponential function to obtain 
the effective half-life. By inversion of this decay function, the point in time was determined when the exemption limit of $10 \mathrm{MBq}$ is expected for a given amount of [ $\left.{ }^{99 \mathrm{~m}} \mathrm{Tc}\right] \mathrm{Tc}-\mathrm{PSMA}-\mathrm{I} \& \mathrm{~S}$.

\section{Measurement of external radiation exposure}

The involved surgeons were equipped with electronic personal dosimeters calibrated in $\mathrm{H}_{\mathrm{p}}(10)$ to determine the actual body dose recorded during the operation. The duration of surgery was recorded to compare the integrated dose results of $\mathrm{H}_{\mathrm{p}}(10)$ with the dose estimates from the patient's time-activity curve.

\section{Estimated maximum number of permissible procedures}

The expected dose rate at a given distance for a given applied activity was calculated using the observed mean effective half-life and the calibration factor of the dose rate meter and with the inverse-square law. For the different dose limits of German categories of occupational radiation exposure per year $(1 \mathrm{mSv}$ for employees without obligatory continuous dose monitoring, $6 \mathrm{mSv}$ for continuously monitored personal in category B, and $20 \mathrm{mSv}$ in category A), the corresponding maximum number of permissible TPRS procedures was calculated.

\section{Statistical analysis}

Continuous measures are reported as mean and standard deviation (SD). Independent samples of continuous measures were compared by Wilcoxon's rank sum test. All calculations were performed with SPSS Version 25 (IBM Corporation, Armonk, NY, USA). P<0.05 was considered significant. 


\section{RESULTS}

\section{Calibration of the dose rate meter from phantom measurements and validation in patients}

The amount of radioactivity $A$ in the cylinder phantom as well as the dose rate $D R$ measurements at time points $t$ are reported in Table 1 together with the resulting calibration factor $K$ and the corresponding measurement uncertainties. From this experiment, a calibration factor $K$ of

$0.013 \pm 0.001 \frac{\mu \mathrm{Sv}}{\mathrm{h} \cdot \mathrm{MBq}}$ was obtained for the dose rate meter. The calibration factor of the instrument from dose rate measurements on 8 patients who have been injected just 1 min before, resulted in a calibration factor $K^{\prime}$ of $0.012 \pm 0.001 \frac{\mu \mathrm{Sv}}{\mathrm{h} \cdot \mathrm{MBq}} . K$ and $K^{\prime}$ do not differ $(\mathrm{p}=0,721)$.

\section{Effective half-life and the time to reach the exemption limit}

A subset of 6 patients underwent repeated dose rate measurements up to 20-27 h p.i. at the time of surgery. The mean effective half-life of $\left[{ }^{99 \mathrm{~m}} \mathrm{Tc}\right] \mathrm{Tc}-\mathrm{PSMA}-\mathrm{I} \& \mathrm{~S}$ in this patient cohort was $4.15 \pm 0.86$ h. Figure 2 shows the resulting dependence of the maximum activity $A_{0}$ to be applied for a remaining activity of $10 \mathrm{MBq}$ as a function of the time between application of the radiopharmaceutical and the scheduled time of surgery. For an operation time of $24 \mathrm{~h}$ after injection of [ $\left.{ }^{99 \mathrm{~m}} \mathrm{Tc}\right] \mathrm{Tc}-\mathrm{PSMA}-\mathrm{I} \& \mathrm{~S}$, an activity of up to $550 \mathrm{MBq}$ can be applied to meet the requirements of radiation protection (staying below the exemption limit).

\section{External radiation exposure of surgeons}

The surgical procedures were performed by a total of 6 involved surgeons. The body dose $\mathrm{H}_{\mathrm{p}}(10)$ measured by electronic personal dosimeters was $4 \pm 1 \mu \mathrm{Sv}$ (range 2-5 $\mu \mathrm{Sv}$ ) with surgery lasting approximately 2 hours. For the observed mean effective half-life of $4.15 \mathrm{~h}$, an applied activity of

$550 \mathrm{MBq}$ and an average distance between surgeon and patient of $0.25 \mathrm{~m}$, a dose rate of $2.08 \frac{\mu \mathrm{Sv}}{\mathrm{h}}$ is calculated. With an average duration of 2 hours, this results in a dose of $4.16 \mu \mathrm{Sv}$ per operation. The dose measured by the dosimeters of the surgeons was $4 \pm 1 \mu \mathrm{Sv}$ and agrees very well with the expected dose estimated from the model.

The maximum numbers of permissible annual TPRS operations for a surgeon according to the dose limits of the different German radiation protection categories are listed in Table 2. For persons not subject to continuous radiation dose monitoring, a maximal annual dose of $1 \mathrm{mSv}$ is allowed. The condition is met, if the surgeon performs up to 241 TPRS operations per year. 


\section{DISCUSSION}

TPRS is a new concept for the treatment of localized relapse from PCa. For some patients, this innovative method offers another therapeutic option. TPRS requires interdisciplinary cooperation of urology and nuclear medicine. Radiation protection issues are involved since there is handling of unsealed radioactivity outside the nuclear medicine department. In our case, it affects 2 separate sites with different hospital providers. The Department of Urology does not have a regulatory permission to handle radioactivities above the exemption limit. Thus, the surgical staff is not subject to radiation protection monitoring.

In order to meet the regulatory requirements for a simplified approval we here estimate the level of occupational radiation exposure. Therefore we performed a calibration of the dose rate meter from phantom measurements with an additional validation in patients. This allowed us to estimate the effective half-life from dose rate measurements to determine the earliest time of surgery. To our best knowledge, there are no reports about such occupational radiation protection aspects for the personnel at present.

In a previous study [8], an average activity of $571 \mathrm{MBq}\left[{ }^{99 \mathrm{~m}} \mathrm{Tc}\right] \mathrm{Tc}-\mathrm{PSMA}-\mathrm{I} \& \mathrm{~S}$ was administered for TPRS starting by average 19.7 hours after injection. This level of applied activity implies the need for full radiation dose monitoring of the involved staff. To safely comply with the $10 \mathrm{MBq}$ limit in the reported setting we can recommend the application of $300 \mathrm{MBq}\left[{ }^{99 \mathrm{~m}} \mathrm{Tc}\right] \mathrm{Tc}-\mathrm{PSMA}-I \& S$ according to our results (Figure 2) to meet the requirements of occupational radiation protection.

With $4.15 \pm 0.86 \mathrm{~h}$, the observed effective half-life of [ $\left.{ }^{99 \mathrm{~m}} \mathrm{Tc}\right] \mathrm{Tc}-\mathrm{PSMA}-\mathrm{I} \& \mathrm{~S}$ is significantly below the physical half-life of ${ }^{99 \mathrm{~m}} \mathrm{Tc}$. A recent study analyzed the internal radiation exposure of [ $\left.{ }^{99 \mathrm{~m}} \mathrm{Tc}\right]$ Tc-HYNIC-PSMA, which exhibits a similar biodistribution as $\left[{ }^{99 \mathrm{~m}} \mathrm{Tc}\right] \mathrm{Tc}-\mathrm{PSMA}-\mathrm{I} \& \mathrm{~S}$ as it is also excreted mainly through the urinary system [11]. By means of whole-body scintigraphies up to $8 \mathrm{~h}$ p.i. the colleagues observed an effective half-life [99m Tc]Tc-HYNIC-PSMA of clearly $<4 \mathrm{~h}$ which is in line with our result of $4.15 \mathrm{~h}$ derived from dose rate measurements. So, the published data give no reason to believe that we may have underestimated the dose values.

The most conservative estimation with an assumed duration of surgery of 4 hours and a distance between surgeon and patient of $0.25 \mathrm{~m}$ still results in a number of 120 permissible operations per surgeon and year. From the point of view of radiation protection, the regular throughput of patients 
qualifying for a salvage surgery procedure is not limited with the usual workplace rotation of surgeons and assistant personnel when performing TPRS.

In some points, radiation protection aspects in TPRS differ from those in sentinel lymph node biopsy (SLNB). Similar to the established practice in SLNB [12], the use of radiation shielding of the surgeon is not practicable and does not seem necessary due to the low expected radiation exposure of surgery staff.

Our study may have some limitations. Even though the analyzed patients showed comparable results in terms of measurement accuracy, the small case number of 6 patients is certainly the main limitation of our study. In order to rapidly establish the method within the legal requirements, we had to waive the examination of a larger patient cohort.

Due to the hardware design of the dose rate meter, the geometric sensitivity may be inhomogeneous and thus affect the dose results. To account for this potential bias, we performed measurements both in a phantom as well as in patients and compared the results. From the strong agreement between the phantom experiment and patient data we see that the dose rate measurements are sufficient to give a reliable estimate of the amount of radioactivity within the patient at any time point. Furthermore, we observed a close correlation of staff exposure measurements by means of dosimeters as compared to the dose estimates from our model using patient-specific parameters.

It should be emphasized that in the present study the effective dose was considered and derived from dose rate estimates to whole-body, but not from partial body doses, especially those of the fingers. In contrast to the situation in SLNB of melanoma, where the injection site is resected, we consider the finger dose in TPRS to be negligible, because after systemic distribution of the tracer no highly concentrated radioactivity is expected in the surgical field. 


\section{CONCLUSION}

With the observed mean effective half-life of $4.15 \mathrm{~h}$ for $\left[{ }^{99 \mathrm{~m}} \mathrm{Tc}\right] \mathrm{Tc}-\mathrm{PSMA}-\mathrm{I} \& \mathrm{~S}$, an average operating time of $2 \mathrm{~h}$ in an average distance between patient and surgeon of $0.25 \mathrm{~m}$ results in a dose of $4.16 \mu \mathrm{Sv}$ per procedure. Thus, approximately 241 operations per surgeon and year are permissible without triggering the full radiation protection monitoring obligations. The TPRS can be performed in a 2-days protocol similarly to the broadly established radioguided SLNB, both organizationally and legally. 


\section{REFERENCES}

1. Siegel RL, Miller KD, Jemal A. Cancer statistics, 2020. CA: a cancer journal for clinicians. 2020;70:7-30. doi:10.3322/caac.21590.

2. Carioli G, Bertuccio P, Boffetta P, Levi F, La Vecchia C, Negri E, et al. European cancer mortality predictions for the year 2020 with a focus on prostate cancer. Annals of Oncology. 2020;31:650-8. doi:10.1016/j.annonc.2020.02.009.

3. von Amsberg G, Merseburger AS. [Treatment of metastatic, castration-resistant prostate cancer]. Der Urologe Ausg A. 2020. doi:10.1007/s00120-020-01187-9.

4. Giesel FL, Hadaschik B, Cardinale J, Radtke J, Vinsensia M, Lehnert W, et al. F-18 labelled PSMA-1007: biodistribution, radiation dosimetry and histopathological validation of tumor lesions in prostate cancer patients. European journal of nuclear medicine and molecular imaging. 2017;44:678-88. doi:10.1007/s00259-016-3573-4.

5. Rauscher I, Eiber M, Jilg CA, Gschwend JE, Maurer T. [PSMA-radioguided surgery in localized recurrent prostate cancer : Current and future aspects]. Der Urologe Ausg A. 2017;56:1823. doi:10.1007/s00120-016-0275-2.

6. Kratochwil C, Bruchertseifer F, Giesel FL, Weis M, Verburg FA, Mottaghy F, et al. 225AcPSMA-617 for PSMA-Targeted alpha-Radiation Therapy of Metastatic Castration-Resistant Prostate Cancer. J Nucl Med. 2016;57:1941-4. doi:10.2967/jnumed.116.178673.

7. Kabasakal L, Toklu T, Yeyin N, Demirci E, Abuqbeitah M, Ocak M, et al. Lu-177-PSMA617 Prostate-Specific Membrane Antigen Inhibitor Therapy in Patients with Castration-Resistant Prostate Cancer: Stability, Bio-distribution and Dosimetry. Molecular imaging and radionuclide therapy. 2017;26:62-8. doi:10.4274/mirt.08760.

8. Maurer T, Robu S, Schottelius M, Schwamborn K, Rauscher I, van den Berg NS, et al. (99m)Technetium-based Prostate-specific Membrane Antigen-radioguided Surgery in Recurrent Prostate Cancer. European urology. 2019;75:659-66. doi:10.1016/j.eururo.2018.03.013.

9. Robu S, Schottelius M, Eiber M, Maurer T, Gschwend J, Schwaiger M, et al. Preclinical Evaluation and First Patient Application of 99mTc-PSMA-I\&S for SPECT Imaging and Radioguided Surgery in Prostate Cancer. J Nucl Med. 2017;58:235-42. doi:10.2967/jnumed.116.178939. 
10. Su HC, Zhu Y, Ling GW, Hu SL, Xu XP, Dai B, et al. Evaluation of 99mTc-labeled PSMASPECT/CT imaging in prostate cancer patients who have undergone biochemical relapse. Asian journal of andrology. 2017;19:267-71. doi:10.4103/1008-682x.192638.

11. Zhang J, Zhang J, Xu X, Lu L, Hu S, Liu C, et al. Evaluation of Radiation dosimetry of (99m)Tc-HYNIC-PSMA and imaging in prostate cancer. Scientific reports. 2020;10:4179. doi:10.1038/s41598-020-61129-5.

12. Bluemel C, Herrmann K, Giammarile F, Nieweg OE, Dubreuil J, Testori A, et al. EANM practice guidelines for lymphoscintigraphy and sentinel lymph node biopsy in melanoma. European journal of nuclear medicine and molecular imaging. 2015;42:1750-66. doi:10.1007/s00259-015-3135-1. 


\section{FIGURE LEGENDS}

\section{Figure 1}

[ $\left.{ }^{99 \mathrm{~m}} \mathrm{Tc}\right]$ Tc-PSMA-I\&S-SPECT/CT (A: coronal, B: axial, C: maximum intensity projection, MIP) and $\left[{ }^{68} \mathrm{Ga}\right] \mathrm{Ga}-\mathrm{PSMA}-11-\mathrm{PET} / \mathrm{CT}$ (D: coronal, E: axial, F: MIP) showing a left iliac lymph node metastasis (red arrow)

\section{Figure 2}

Maximum activity $\mathrm{A}_{0}$ to be applied for a remaining activity of $10 \mathrm{MBq}$ as a function of the time $\mathrm{t}$ until planned start of surgery, resulting from the observed mean effective half-life of [ $\left.{ }^{99 \mathrm{~m}} \mathrm{Tc}\right] \mathrm{Tc}-$ PSMA-I\&S of $4.15 \mathrm{~h}$. 


\section{TABLE LEGENDS}

\section{Table 1}

Determination of the calibration factor from dose rate measurements on a cylinder phantom

\section{Table 2}

Maximum number of annual TPRS operations of a surgeon assuming a mean duration of 1, 2, 3 or $4 \mathrm{~h}$, respectively, in a mean distance of $0.25,0.5,0.75$ or $1 \mathrm{~m}$ to the patient before reaching the dose limits of the different German radiation protection categories 


\section{DECLARATION}

\section{Funding}

There was no funding for this study.

\section{Conflicts of interest/Competing interests}

The authors declare, that they have no conflict of interest or competing interests.

\section{Ethics approval}

This retrospective study was performed in accordance with all relevant guidelines and regulations. Based on the retrospective nature and a fully anonymized set of clinical data the need of an informed consent was waived by the institutional authorities.

\section{Consent to publication}

All authors agreed with the content and gave explicit consent for publication. All authors obtained consent from the responsible authorities at the institutes where the work has been carried out.

\section{Availability of data and material}

All individual de-identified data will be shared upon request.

\section{Code availability}

All software applications are mentioned in the section "Material and methods".

\section{Authors' contributions}

Conceptualization and design: Daniel Schmidt, Jirka Grosse, Dirk Hellwig

Methodology: Daniel Schmidt

Formal analysis and investigation: Daniel Schmidt

Figures and visualization: Daniel Schmidt

Writing - original draft preparation: Daniel Schmidt, Jirka Grosse, Dirk Hellwig

Writing - review and editing: Jutta Moosbauer, Roman Mayr, Max Burger, Dirk Hellwig

Supervision: Dirk Hellwig

All authors read and approved the final manuscript. 
Figures
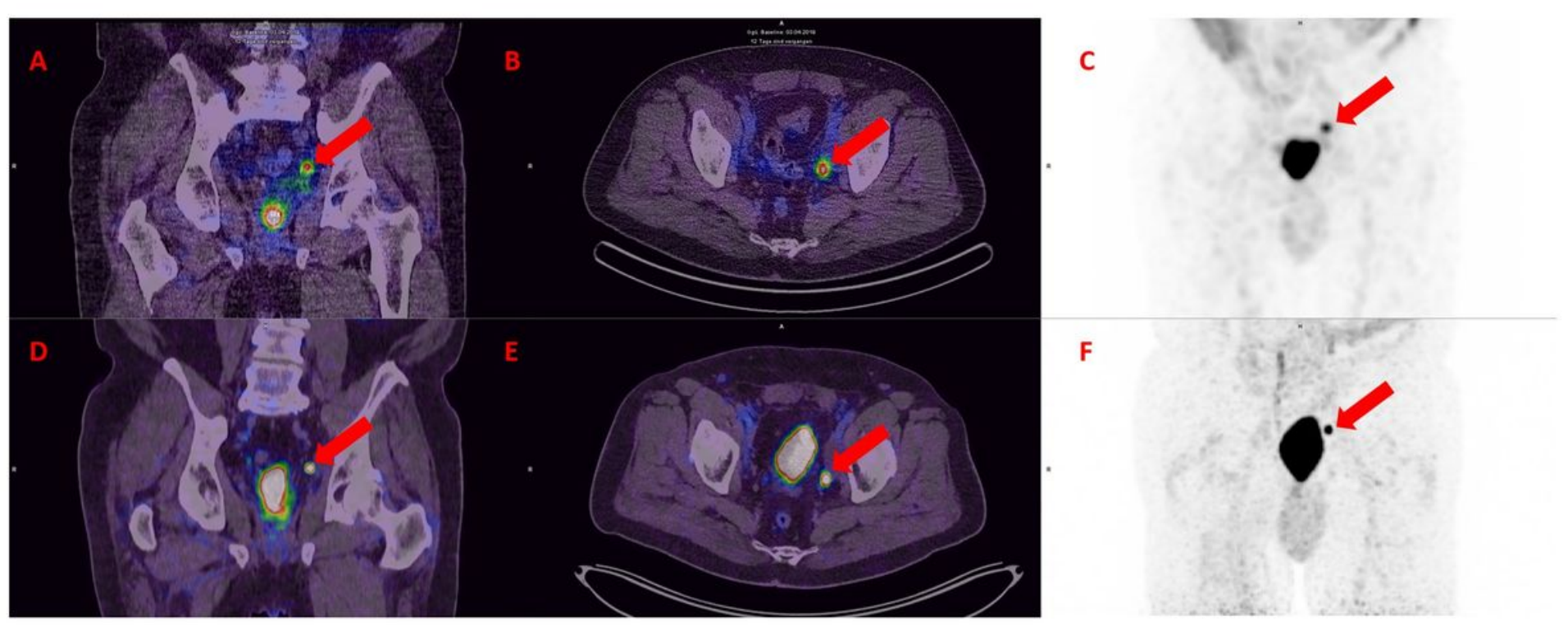

Figure 1

[99mTc]TC-PSMA-I\&S-SPECT/CT (A: coronal, B: axial, C: maximum intensity projection, MIP) and [68Ga]Ga-PSMA-11-PET/CT (D: coronal, E: axial, F: MIP) showing a left iliac lymph node metastasis (red arrow) 


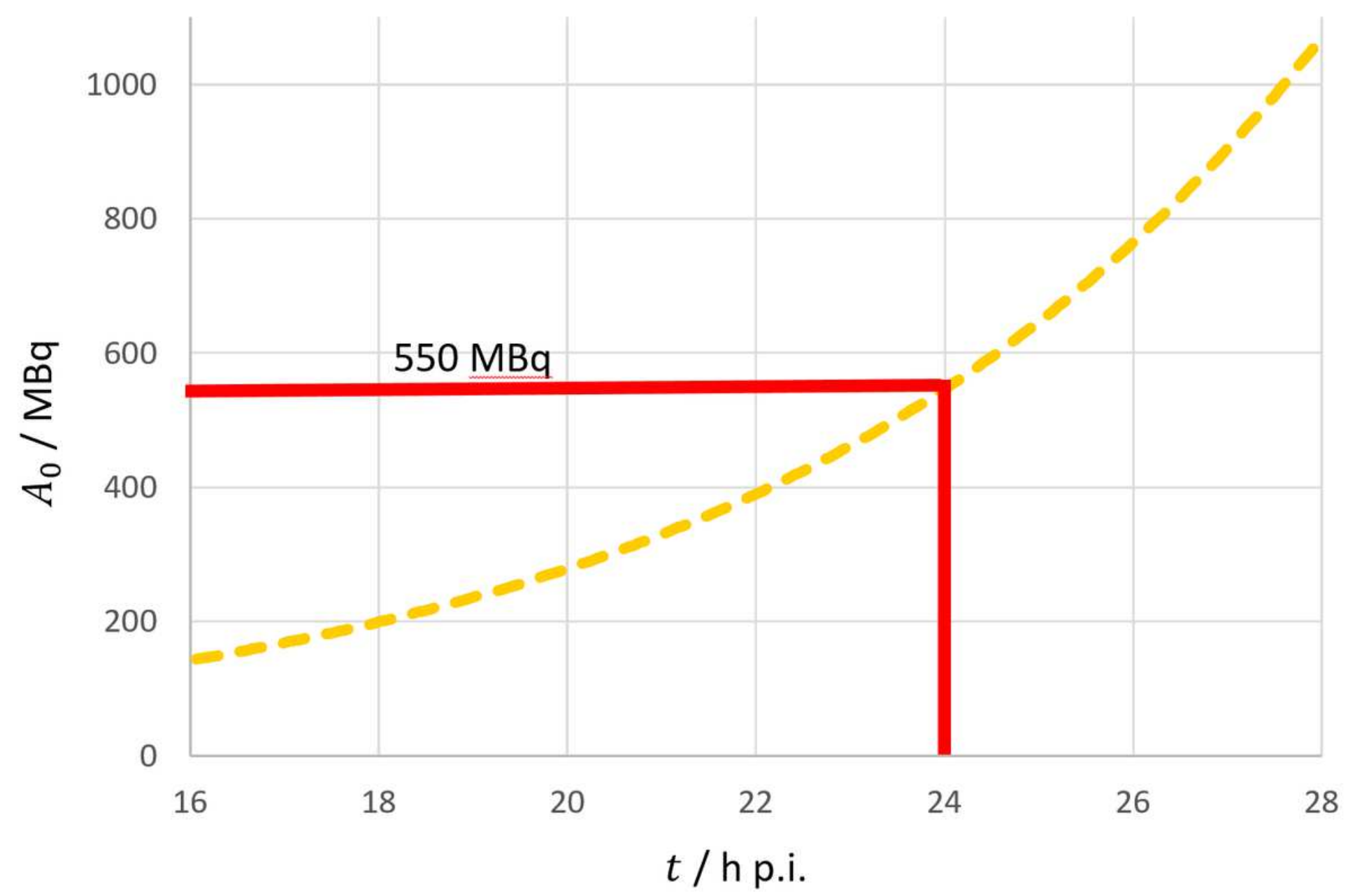

Figure 2

Maximum activity $\mathrm{A} 0$ to be applied for a remaining activity of $10 \mathrm{MBq}$ as a function of the time $t$ until planned start of surgery, resulting from the observed mean effective half-life of [99mTc]Tc-PSMA-I\&S of $4.15 \mathrm{~h}$.

\section{Supplementary Files}

This is a list of supplementary files associated with this preprint. Click to download.

- Table1.docx

- Table2.docx 